\title{
Computer Aided Design of Transformer Station Grounding System Using CDEGS Software
}

\author{
S. Nikolovski, T. Barić
}

This paper presents a computer-aided design of a transformer station grounding system. Fault conditions in a transformer station can produce huge damage to transformer station equipment if the grounding system is not designed properly. A well designed grounding system is a very important part of the project for transformer station design as a whole. This paper analyses a procedure for transformer grounding system design and spatial distribution of touch and step voltage on the ground surface level, using the CDEGS (Current Distribution Electromagnetic Interference Grounding and Soil Structure Analysis) software. Spatial distribution is needed for checking and finding dangerous step and touch voltages above and around the transformer station. Apparent earth resistivity data is measured and analyzed using the RESAP module of the CDEGS software. Because of the very high current flow into the grounding system during a single line to ground fault or a three phase fault in the transformer station, very high and dangerous potentials can be induced on the metallic structures including the fence, which can cause dangerous situations for people and animals near the station and for the personnel inside the station. The PLOT module of CDEGS is used to view the results of the scalar potential, step and touch voltage on the surface. Graphic displays include equipotent contour lines and potential profiles (gradients) in $3 D$ and $2 D$ perspective and apparent soil resistivity ( $\Omega m$ ) versus inter electrode spacing $(m)$. The results of alternative grid designs may be displayed simultaneously for the purpose of comparison.

Keywords: computer-aided design, substation, grounding grid, soil, safety, touch and step voltage.

\section{Introduction}

With ever increasing fault current levels in today's interconnected power systems it is necessary to ensure very low grounding resistance of transformer stations. In order to get the best techno-economic solution in the design of grounding systems for given safety criteria (IEEE, IEC, or some other National standard) only computer-aided design can give an optimal and fast solution of the given task. Low grounding resistance and acceptable distribution of touch and step voltages (as uniform as possible) for a high fault current level can be simultaneously achieved only by using a grid-grounding system.

The task for designers of grounding systems is to arrange a buried metallic conductor with adequate equivalent radii to achieve the safety criteria. The safety criteria in this paper are based on IEEE Std. 80, 188 editions and IEC 479-1, 1984 and some Croatian safety requirements. Another task is to check some existing grounding grids for an increased fault current level, i.e., to determine the fault current level which satisfies the safety criteria. A typical grounding grid is designed and simulated using the HIFREQ module. A design problem is

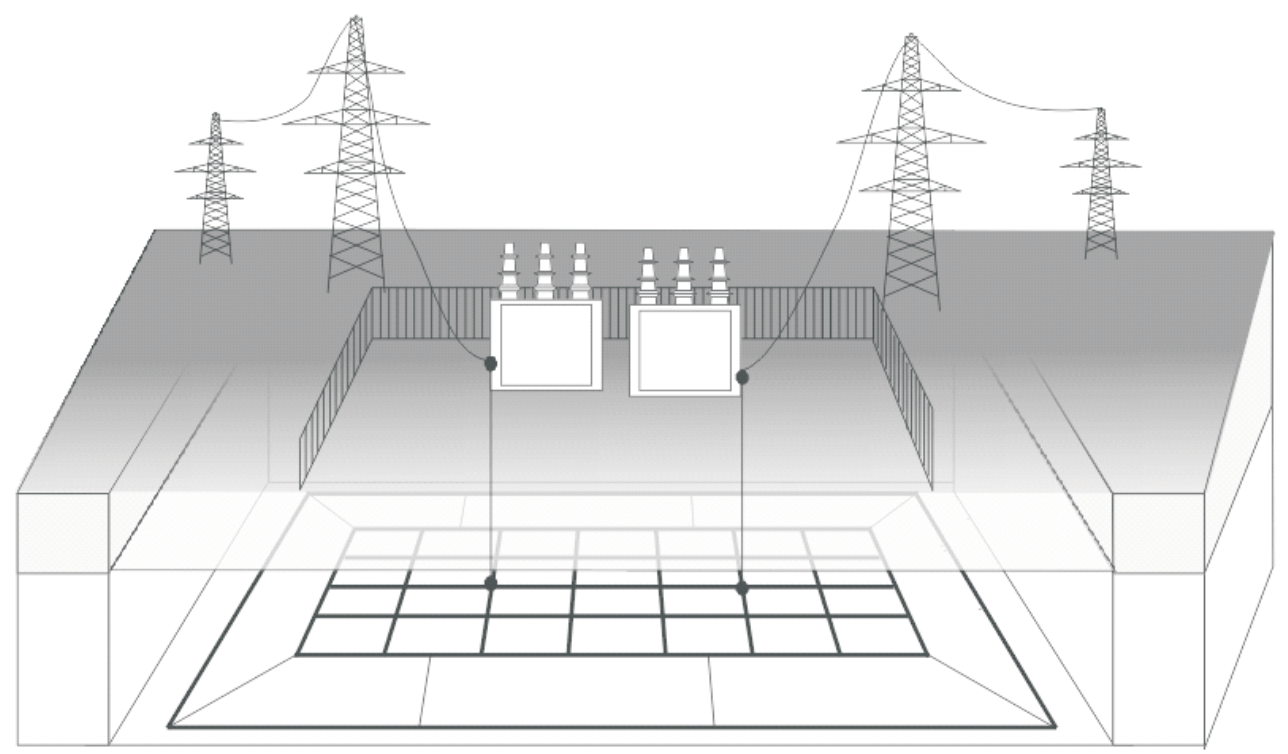

Fig. 1: Simplified geometrical model of a transformer station grounding system and the conducting part 
described and verified for several (110/35/10 kV) transformer substations: "TS Našice”, "TS Osijek", and "TS Valpovo". These transformer substations are in the eastern part of the Croatian power system. Due to their operational time, TS are exposed to potential fault conditions. In this sample case a single line to ground fault is relevant for the grounding design. According to the technical specifications, single line to ground fault currents entering the grounding system of these transformer substations are in the order of 6 to $10 \mathrm{kA}$. The analysed grounding system consists of a $110 \times 80 \mathrm{~m}$ grounding grid with 20-30 additional grounding rods of 3 m length. When this happens, the potentials on the surfaces inside the TS and nearby are on a much higher level than the reference point away from TS.

\section{Mathematical expression of the electromagnetic field}

The conductor network is subdivided into small segments. This allows the thin-wire approximation to be used, which in turn enables us to use linear current sources. The method used to obtain the scalar potential and the electromagnetic field in the frequency domain is described by using the following 3 equations. Maxwell's equations can be used to describe electric field $\boldsymbol{E}$ and magnetic field $\boldsymbol{H}$ in terms of scalar $\varphi$ and vector potential $\boldsymbol{A}$ :

$$
\begin{aligned}
& \varphi=\frac{1}{-\mu \theta} \nabla \cdot A \\
& \boldsymbol{E}=-\mathrm{j} \omega \boldsymbol{A}+\nabla \varphi \\
& \boldsymbol{H}=\frac{1}{\mu} \cdot \nabla x \mathrm{~A} \\
& \theta=\sigma+\mathrm{j} \omega \varepsilon
\end{aligned}
$$

where: $\theta \quad$ complex conductivity of the medium,

$$
\begin{array}{ll}
\mu & \text { permeability of the medium, } \\
\sigma & \text { conductivity of the medium, } \\
\varepsilon & \text { permittivity of the medium. }
\end{array}
$$

\section{Example of transformer station grounding design}

Through there is no conventional rule in designing transformer station grounding, some guidelines can be suggested on the basis of this example using CDEGS, as follows. The first step in the design of transformer station grounding is to determine appropriate soil model, for predicting the effect of the underlying soil characteristics on the performance of the grounding system. From the resistivity measurement data obtained using arbitrarily spaced 4-electrode configuration methods (including Wenner or Schlumberger methods), the RESAP module determines the equivalent soil layers. The earth layers may be vertical or horizontal (a one-layer, a two-layer or a multi-layer soil model). In this study, soil resistivity is measured by a 4-point measurement (Schlumberger method). The influence of seasonal variations in the last several years on soil resistivity is estimated indirectly using statistical data collected in the course of preventive measurements of the grounding impedance by a local utility. According to this simplified method, $100 \Omega \mathrm{m}$ soil resistivitiy can be assumed as the worst soil top layer resistivity. The RESAP program interprets the measured apparent earth resistivity (or resistance) data to determine the equivalent earth structure model that is necessary for analysing the grounding systems. The measured soil resistivity data is shown in Table 1.

Measured data entered in the RESAP (computations) window is shown in Fig 2. The 4-point Schlumberger measurement method with necessary data for calculating soil resistivity is also presented. The RESAP module calculates the resistivity using the following generalised equation:

$$
\rho=2 \pi R\left[\frac{1}{S_{e 1}}+\frac{1}{S_{e 2}}+\frac{1}{S_{e 1}+S_{i}}+\frac{1}{S_{e 2}+S_{i}}\right]
$$

where: $S_{e 1} \quad$ distance between current C1 and potential P1 electrodes,

$$
\begin{array}{ll}
S_{e 2} \quad \begin{array}{l}
\text { distance between current C2 and potential P2 } \\
\text { electrodes, }
\end{array} \\
S_{i} \quad \text { distance between potential electrodes, } \\
\rho \quad \text { resistivity of soil, } \\
R \quad \text { measurement of soil resistance. }
\end{array}
$$

The apparent soil resistivity in an $\Omega \mathrm{m}$ (versus inter-electrode spacing in $\mathrm{m}$ ) relevant in this study is shown in Fig. 3. According to Fig 3., after $2 \mathrm{~m}$ below the surface the apparent resistivity becomes practically constant. Fig. 3. represents one measurement series, which cannot represent the resistivity during seasons. It is useful to remember that current depth penetration in soil depends on current electrode spacing. It is therefore useful to perform some additional measurements with a very close inter-electrode to get a better interpolation of the soil layer just below the surface, since the apparent resistivity has changed rapidly in one layer.

The computed soil resistivity and layer thickness for a 2-layer soil model are given by the text report, and are shown below in the computer generated report.

Table 1: Measurement data

\begin{tabular}{|lc|c|c|c|c|c|c|c|c|c|c|}
\hline Measurement number & & 1. & 2. & 3. & 4. & 5. & 6. & 7. & 8. & 9. & 10. \\
\hline \multirow{2}{*}{ Distance between electrode $[\mathrm{m}]$} & $S_{e}$ & 0.25 & 0.75 & 1.25 & 1.75 & 2.25 & 2.75 & 3.25 & 3.75 & 4.75 & 5.75 \\
\cline { 3 - 38 } & $S_{i}$ & 0.50 & 0.50 & 0.50 & 0.50 & 0.50 & 0.50 & 0.50 & 0.50 & 0.50 & 0.50 \\
\hline Measured resistance $[\Omega]$ & $R$ & 42.200 & 6.130 & 2.530 & 1.372 & 0.906 & 0.604 & 0.446 & 0.338 & 0.214 & 0.148 \\
\hline
\end{tabular}




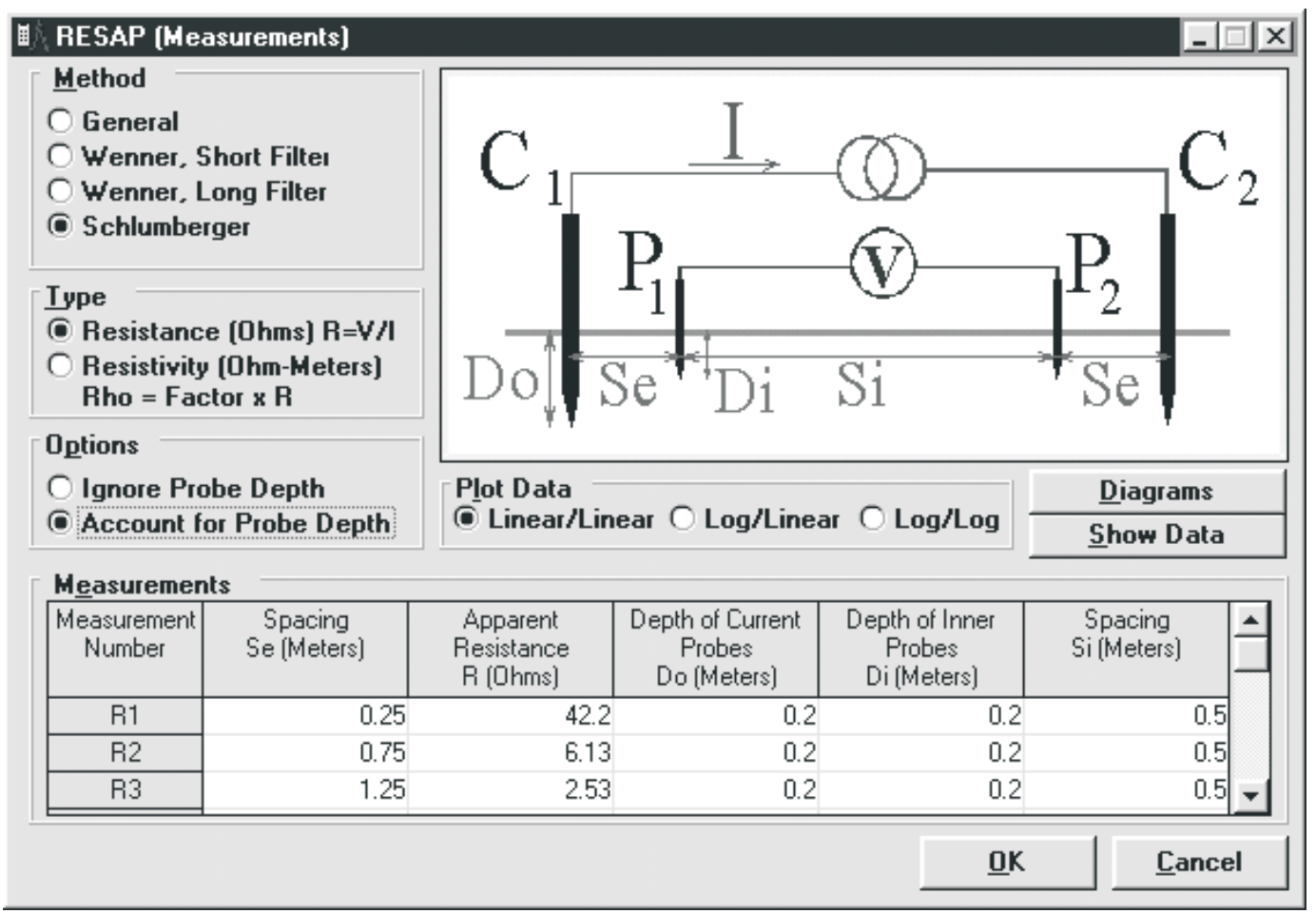

Fig. 2: RESAP (computations) window with measurement data relevant in this study

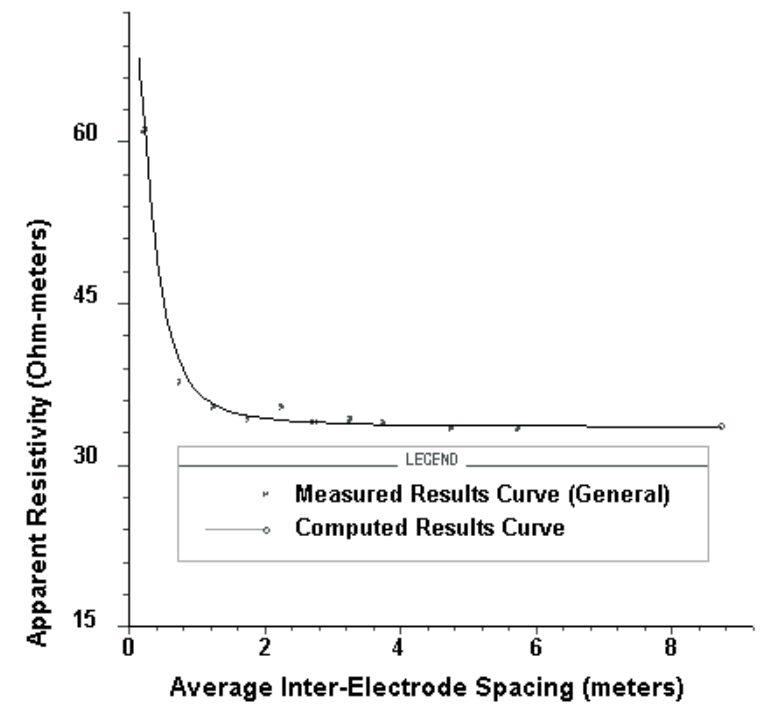

Fig. 3: Apparent soil resistivity versus inter-electrode spacing

\section{R E S I S T I V IT Y \\ (SYSTEM INFORMATION SUMMARY)}

System of Units:

Meters

Soil Type Selected: Multi-Layer Horizontal

RMS error between measured and calculated: $2.08052 \%$

resistivities

\begin{tabular}{|c|c|c|c|c|}
\hline $\begin{array}{c}\text { Layer } \\
\text { Number }\end{array}$ & $\begin{array}{c}\text { Resistivity } \\
(\Omega \mathrm{m})\end{array}$ & $\begin{array}{c}\text { Thickness } \\
(\mathrm{m})\end{array}$ & $\begin{array}{c}\text { Coefficient } \\
(\text { p.u. })\end{array}$ & $\begin{array}{c}\text { Contrast } \\
\text { Ratio }\end{array}$ \\
\hline 1 & infinite & infinite & 0.0 & 1.0 \\
\hline 2 & 78.17224 & 0.2106636 & -1.0000 & $0.78172 \mathrm{E}-18$ \\
\hline 3 & 33.55941 & infinite & -0.39929 & 0.42930 \\
\hline
\end{tabular}

(C) Czech Technical University Publishing House http://ctn.cvut.cz/ap/
Soil layer resistivity calculated by RESAP is the initial data for a further study. From the computer generated report for soil resistivity, a two-layer soil model is made, which gives satisfactory solutions in most cases including this study. The data entered in the second module HIFREQ is shown in Fig. 4. Data for relative permeability and permittivity is irrelevant in this study.

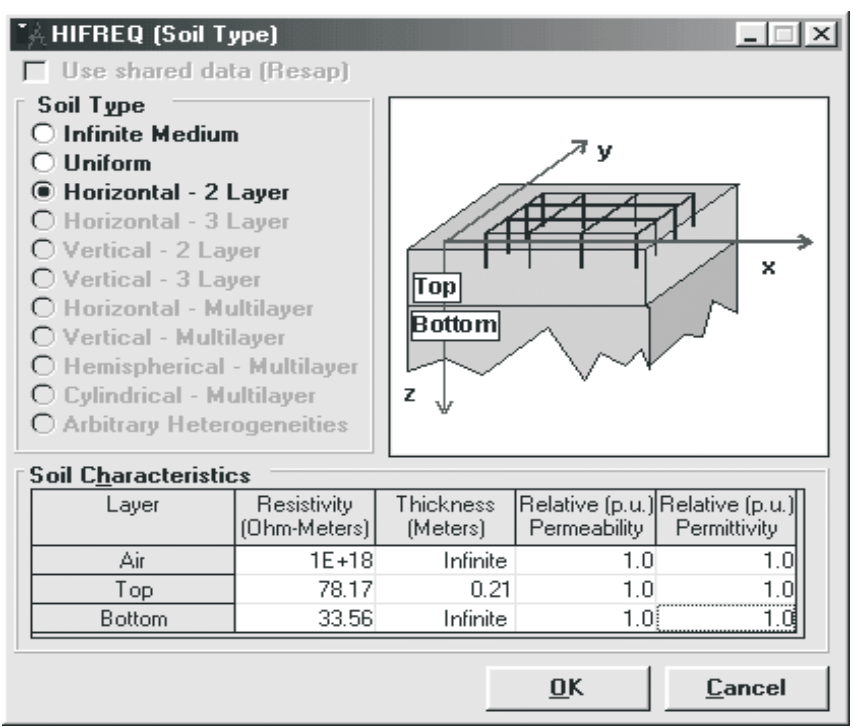

Fig. 4: A HIFREQ (Soil type) window is used to specify the type of soil

The grounding system described in this study consists of a $110 \times 80 \mathrm{~m}$ grounding grid plus an additional ring $5 \mathrm{~m}$ away from the grid. The grounding system is buried 0.8 meters below the transformer station surface. The ground fault current in this study is assumed to be $7 \mathrm{kA}$. The grounding grid con- 


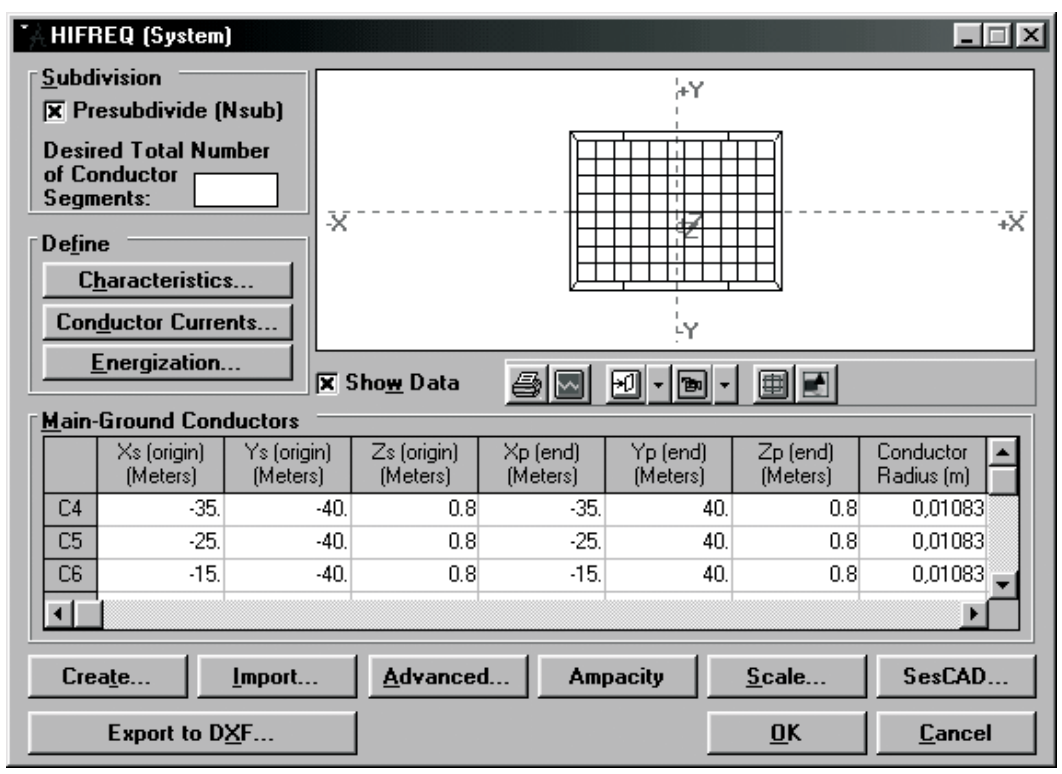

Fig. 5: Geometry of the grounding grid with data relevant in this study

sists of rectangular cross section $\mathrm{Fe} / \mathrm{Zn}$ conductors $(30 \times 4 \mathrm{~mm})$ with an equivalent radius equal to $10.83 \mathrm{~mm}$. A geometrical position of the grounding grid is shown in Fig 5 .

This first iteration is without additional rods, e.g., the grounding could consist of the grounding grid, with rods and an additional ring around it at the same depth. Division of the grid into smaller windows is not appropriate in the first design iteration. The result of simulation is presented in the following figures. First, a 3D plot of scalar potential at the surface of TS is shown in Fig. 6. The scalar potential peaks correspond to the nodes of the grid, e.g., the valleys correspond to the centre of the windows in the grid (see Fig. 6). This $3 \mathrm{D}$ view is useful in visualisation of the scalar potential shape. This shape should look as uniform as possible. Fig. 7 shows the reach touch voltages in $3 \mathrm{D}$ view with references to the worst system grounding potential rise (GPR). Fig. 7 shows that the reach touch voltages are worst in the corners of TS, and additional measures must be taken to decrease these voltages below safety limits.

Since a 3D plot loses some detailed information about scalar potential or step/touch voltages, an additional investigation must be made for suspicious areas or profiles. An additional inspection can be made in a 2D view for arbitrary profiles (see Fig. 8). Reach touch voltages for the worst system GPR at the surface of TS, for three profiles with numbers 1, 3 and 100, are shown in Fig. 8.

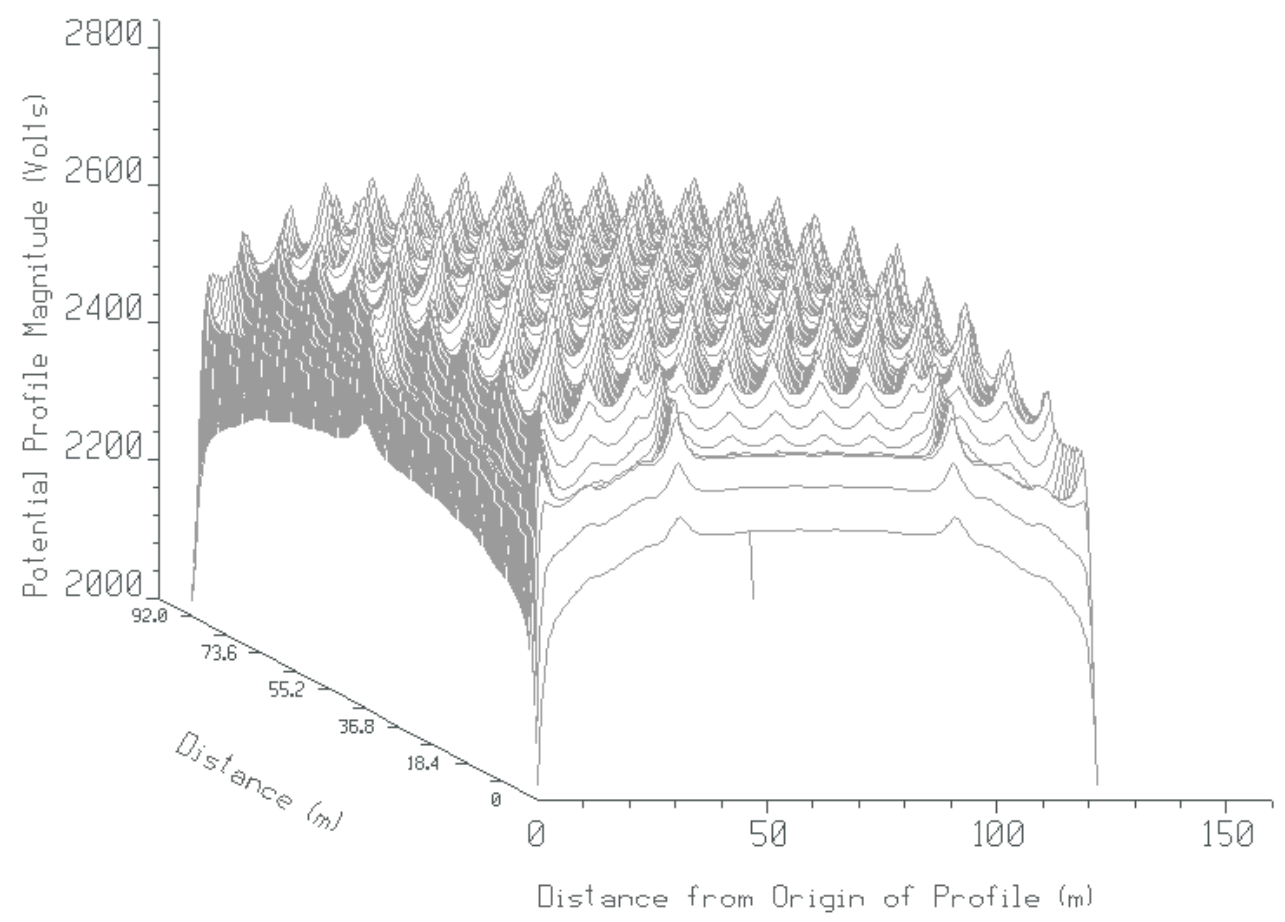

Fig. 6: Scalar potential at the surface of TS 


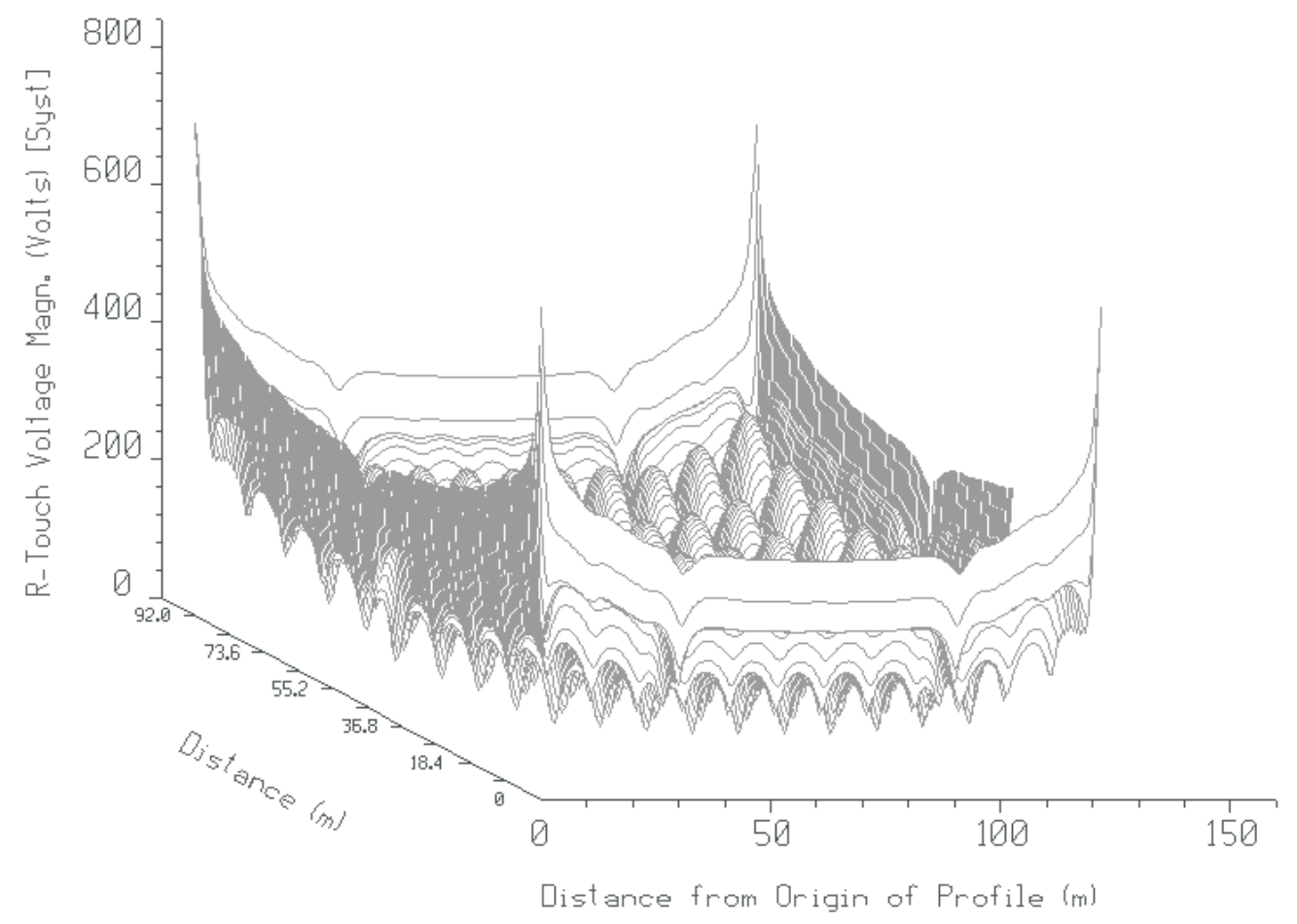

Fig. 7: Reach touch voltages in 3D plot, corresponding to the worst system GPR

A similar investigation can be made in a $2 \mathrm{D}$ view, as shown in Fig. 9. These 2D contour views show lines of the same reach touch voltages with the superposed grid.
The safety limits for step and touch voltages are generated by CDEGS according to user defined standards. Several different standards can be chosen (IEEE, IEC or some national
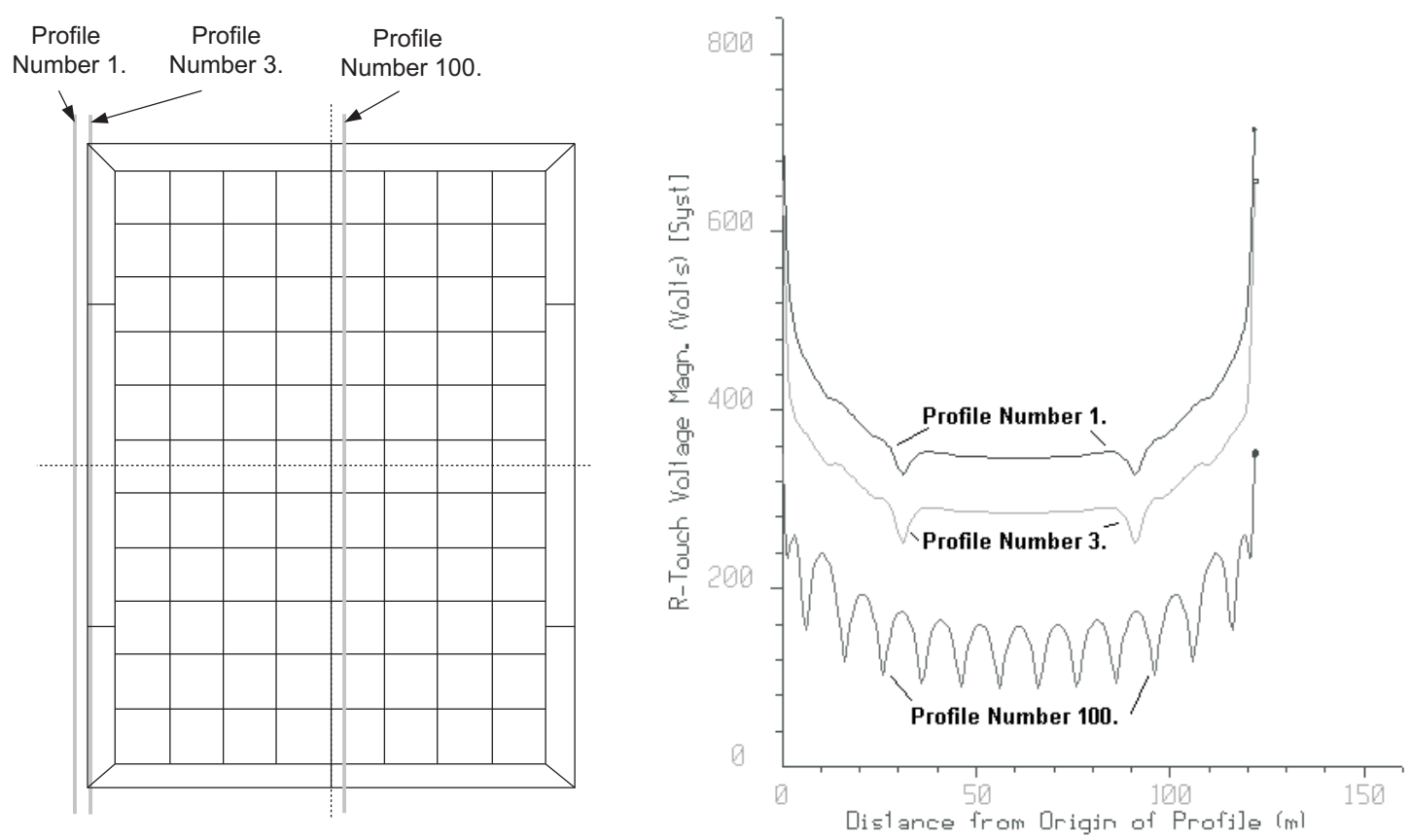

Fig. 8: Reach touch voltages/worst system GPR 


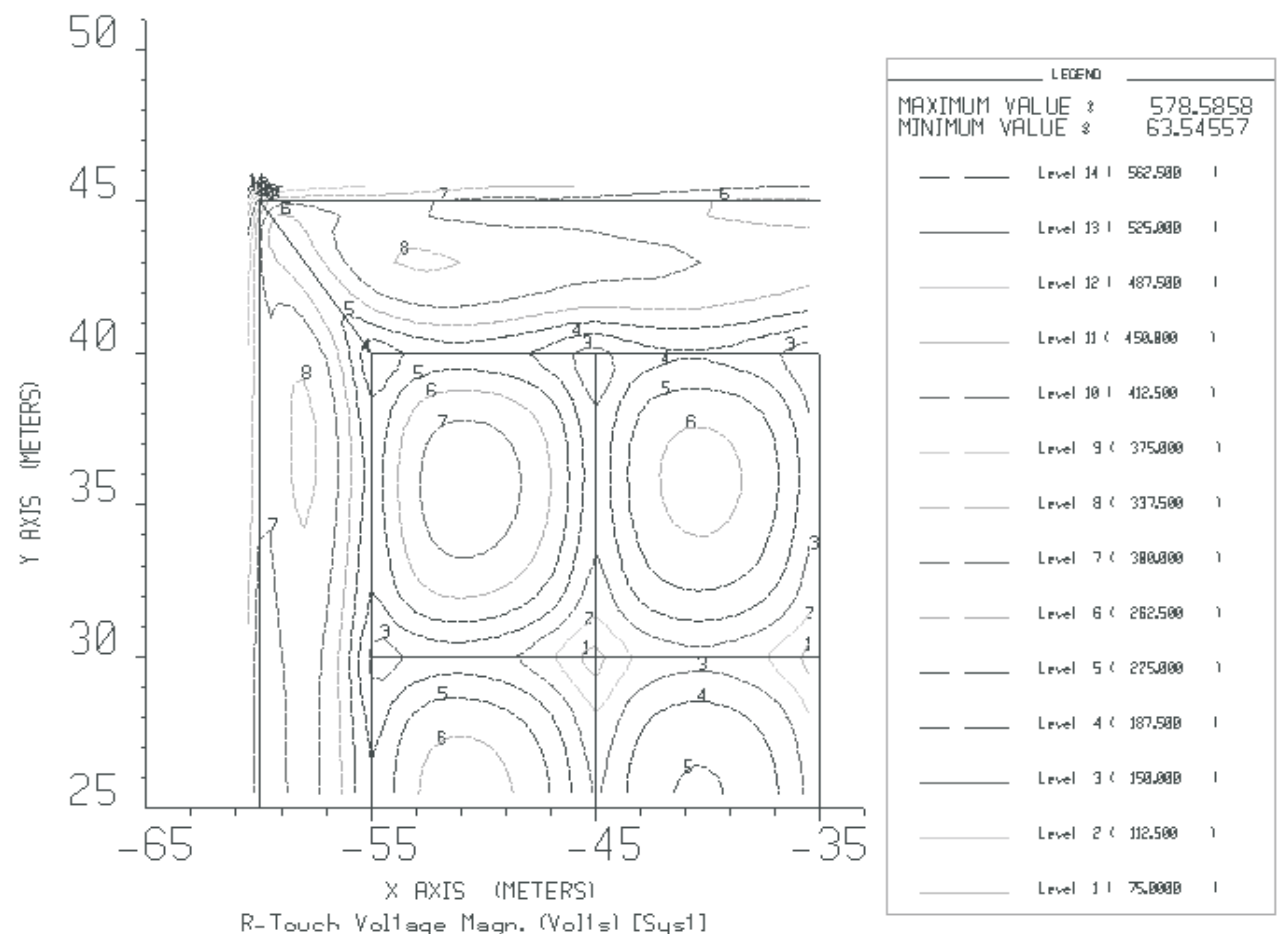

Fig. 9: Reach touch voltages/ worst system GPR contour plot

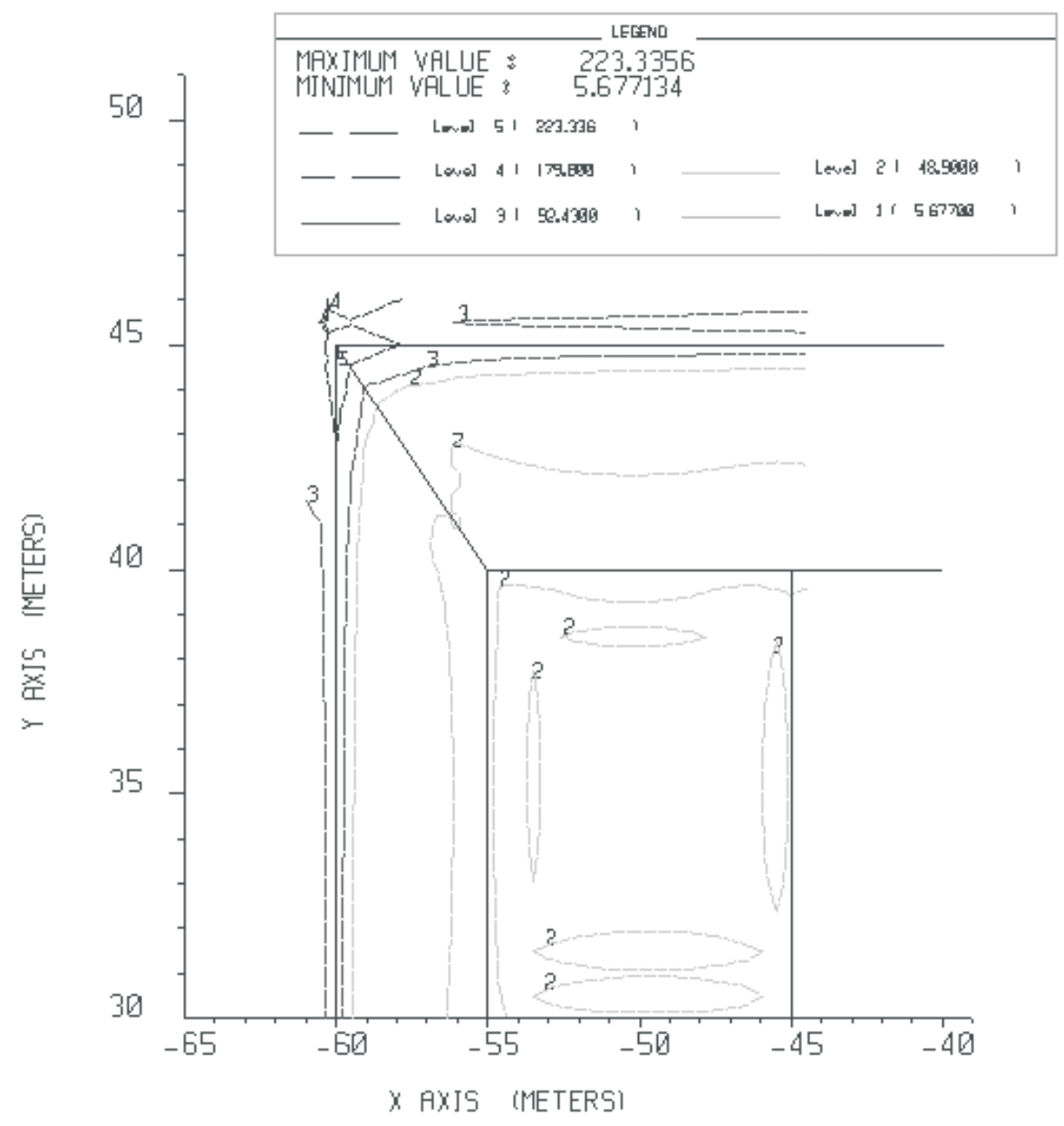

Fig. 10: Reach step voltages/ worst system GPR contour plot 


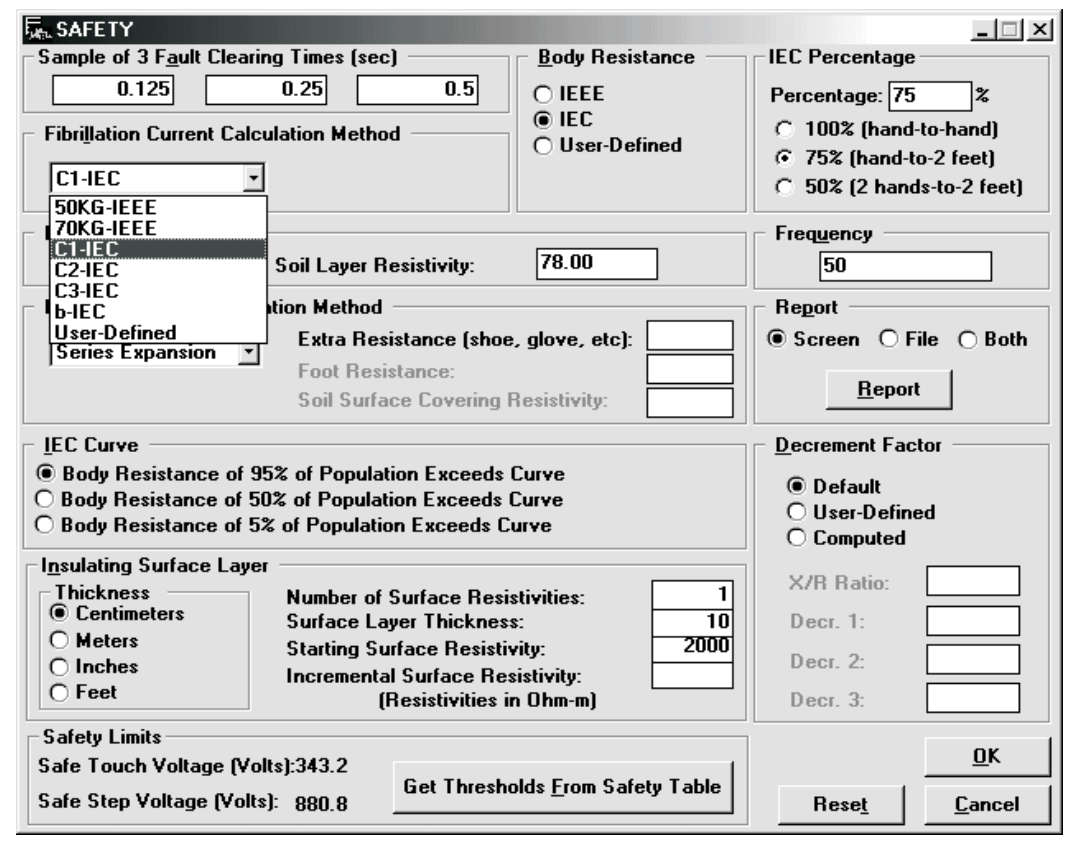

Fig. 11: Window for Safety assessment limits with data relevant in this study

standard). In this study the fibrillation current is calculated according to the C1-IEC standard and body resistance for $95 \%$ population, see Fig. 11. If crushed rock is used to improve the safety limits for step and touch voltage, it can be included and entered in the window.

\begin{tabular}{|c|c|c|c|c|c|c|}
\hline \multicolumn{7}{|c|}{ Safety Calculation Table } \\
\hline & \multicolumn{6}{|c|}{\begin{tabular}{|c|} 
Fault Clearing Time \\
\end{tabular}} \\
\hline & \multicolumn{2}{|c|}{$0.125 \mathrm{sec}$} & \multicolumn{2}{|c|}{\begin{tabular}{|c|}
$0.25 \mathrm{sec}$ \\
\end{tabular}} & \multicolumn{2}{|c|}{$0.5 \mathrm{sec}$} \\
\hline $\begin{array}{c}\text { Surface Layer } \\
\text { Resistivity }\end{array}$ & \begin{tabular}{|l|} 
Step [v] \\
Voltage
\end{tabular} & \begin{tabular}{|c|} 
Touch (v) \\
Voltage
\end{tabular} & $\begin{array}{l}\text { Step [v] } \\
\text { Voltage }\end{array}$ & $\begin{array}{c}\text { Touch [v] } \\
\text { Voltage }\end{array}$ & $\begin{array}{l}\text { Step [v] } \\
\text { Voltage }\end{array}$ & \begin{tabular}{|c|} 
Touch (v) \\
Voltage
\end{tabular} \\
\hline 2000.0 & 2538.1 & 815.1 & 2265.5 & 731.0 & 943.9 & 315.1 \\
\hline
\end{tabular}

Fig. 12: Computed safety Step and Touch Voltage for an additional layer (rocks) at the surface of TS

\section{Conclusion}

Manual calculation of the equivalent resistance of composite grounding can lead to incorrect results if the mirroring effect is not taken into account. Because of the large geometric structure of a grid, all additional metallic conductors such as rods, rings and another additional structures can influence each other. This gives advantages to computer design, especially to specialised programs that take into account all physical phenomena. Since TS is a restricted area for non-operating personnel (civilian personnel), it is protected by a metallic fence. Attention must be taken to prevent the rise in the potential of fence due to fault conditions in TS. Fatal accidents caused by dangerous touch voltages when someone touches the fence can be avoided by placing an additional buried ring conductor outside TS, one meter away from the fence, which is in galvanic contact with the main grounding but not with the fence. This additional ring is added in this study. The main purpose of this additional ring conductor is to shape the electric potential at the surface around the fence.

\section{References}

[1] Ma J., Dawalibi F. P.: "Modern Computational Methods for the Design and Analysis of Power System Grounding." Proceedings of the 1998 International Conference on Power System Technology, Beijing, August 18-21, 1998, p. 122-126.

[2] Dawalibi F. P., Barbeito N.: "Measurements and Computations of the Performance of Grounding Systems Buried in Multilayer Soils." IEEE Transactions on Power Delivery, Vol. 6, No. 4, October 1991, p. 1483-1490.

[3] Nikolovski S., Fortin S.: "Lightning Transient Response of $400 \mathrm{kV}$ Transmission Tower with Associated Grounding System.” EMC 1998 Roma International Symposium on Electromagnetic Compatibility, Rome, Italy, September 14, 1998.

[4] Nikolovski S., Fortin S.: "Frequency Domain Analysis of 110/35 kV Transformer Station Grounding System Subject to Lightning Strike." Proceedings of the IEEE PowerTech '99 Conference, Budapest, Hungary, August 29-September 2, 1999.

Srete Nikolovski Ph.D

e-mail: Srete.Nikolovski@etfos.hr

Tomislav Barić B.Sc.

e-mail: Tomislav.Baric@etfos.hr

Power System Department

Faculty of Electrical Engineering Osijek

K. Trpimira 2B

31000 Osijek, Croatia 